\title{
CT and Clinical Predictors of Fatigue at One Month after Stroke
}

\author{
Mansur A. Kutlubaeva,b Susan D. Shenkin ${ }^{b, c}$ Andrew J. Farrall ${ }^{b}$ \\ Fiona H. Duncan ${ }^{a}$ Susan J. Lewis ${ }^{a}$ Carolyn A. Greig ${ }^{a}$ Martin S. Dennis ${ }^{b}$ \\ Joanna M. Wardlaw ${ }^{b, c}$ Alasdair M.J. MacLullicha, ${ }^{a}$ Gillian E. Mead ${ }^{a, d}$ \\ ${ }^{a}$ Geriatric Medicine, ${ }^{b}$ Clinical Neurosciences, ${ }^{c}$ Centre for Cognitive Ageing and Cognitive \\ Epidemiology, and ${ }^{\mathrm{d} C e n t r e}$ for Clinical Brain Sciences, University of Edinburgh, Edinburgh, UK
}

Key Words

Stroke recovery $\cdot$ Fatigue $\cdot \mathrm{CT} \cdot$ White matter disease $\cdot$ Atrophy

\section{Abstract}

Background: Fatigue is a common and distressing consequence of stroke, and the aetiology of post-stroke fatigue (PSF) is poorly understood. It is unclear whether chronic brain changes [cerebral atrophy and white matter lesions (WML)], stroke lesion location or certain clinical features are related to its development. The aim of this study was to identify, in patients with acute stroke, whether features in different brain regions on routine $C T$ imaging or routinely collected clinical features predicted PSF at 1 month. Methods: In total, 107 patients (62\% male) with acute ischaemic or haemorrhagic stroke were assessed for fatigue (Fatigue Assessment Scale), anxiety and depression (Hospital Anxiety and Depression Scale) at 1 month. Admission brain $\mathrm{CT}$ was rated using a structured scoring system for (i) severity of atrophy and (ii) severity of WML in different regions of the brain, and (iii) site of acute and previous vascular lesions. Results: Cerebral atrophy of mild or greater severity was present in 84 patients (77.5\%) and WML of mild or greater severity was present in 54 patients (50.5\%) in at least one of the evaluated brain regions. There was no association between PSF and severity of atrophy or WML, or presence of acute or previous vascular lesions. We used the Oxfordshire Community Stroke Project (OCSP) classification to explore the possible influence of lesion location because a minority of the patients (37.4\%) had visible acute lesions. Fatigue scores were higher in patients with clinically diagnosed posterior strokes $(p=0.046)$, in females $(p=0.05)$ and in those with higher depression and anxiety scores $(\rho=0.52 ; p<0.001$ and $\rho=0.49 ; p<0.001$, respectively). Structural CT variables were not significant predictors of fatigue (log FAS) in a linear 
regression which controlled for age, sex, pre-stroke fatigue, OCSP classification, depression and anxiety. The significant predictors of fatigue were depression $(\beta=0.30 ; p=0.007)$ and anxiety $\left(\beta=0.28 ; p=0.013\right.$; adjusted $R^{2}=0.254$ ). Stroke subtype (according to the OCSP classification) was marginally predictive $(\beta=0.17 ; p=0.05)$ and sex was not statistically significant $(\beta=0.15 ; p=0.08)$. Conclusions: Features on routine post-stroke CT do not appear to associate with fatigue at 1 month. However, clinically diagnosed posterior strokes as well as female gender, anxiety and depression may be linked with fatigue. Therefore, clinical vigilance rather than $\mathrm{CT}$ features should be used to predict fatigue early after stroke. Further research is needed in this area to establish whether biological mechanisms underlie the development of PSF.

Copyright (C) 2013 S. Karger AG, Basel

\section{Introduction}

Fatigue is a common and distressing consequence of stroke [1,2]. Its aetiology is poorly understood [1]. Fatigue starts early after stroke [3] and is more common in patients with minor stroke than transient ischaemic attack, indicating that the stroke lesion itself may contribute to its development [4]. Some studies have reported an association between poststroke fatigue (PSF) and stroke lesion location. Fatigue 3 months after stroke has been shown to be associated with acute infarcts in the basal ganglia detected on MRI ( $n=334)$ [5], and fatigue 2 months after stroke has been demonstrated to be more common in patients with infratentorial lesions detected on CT or MRI $(n=108)$ [3]. Two studies found a relationship between fatigue and lesions in the posterior circulation territory, defined as brainstem strokes [6] or basilar artery infarctions [7]. These studies included patients relatively late after stroke (time elapsed since the stroke varied from 3 months to 12 years) and one of them included only young patients after first-ever stroke [7]. However, some studies found no association between PSF and lesion location, which could be due to the sample size, use of different neuroimaging methods (MRI or CT), broad categories for defining lesion location, different methods for assessing fatigue and different inclusion/exclusion criteria such as timing after stroke, exclusion of those with depression, etc. [1].

The presence of brain changes associated with ageing [atrophy and white matter lesions (WML)] may also contribute to the development of fatigue. Previous studies have found conflicting results. One study revealed an association between the overall burden of WML on CT and PSF but did not assess atrophy [8]. Fatigue was assessed using a postal questionnaire with a relatively low response rate, between 185 and 756 days after stroke. Another study considered both atrophy and WML, using either CT or MRI, and did not find any association with fatigue [3]. Fatigue was assessed using the Checklist Individual Strength at 2 months and 1.5 years after stroke.

Significant fatigue has been reported in over $50 \%$ of patients on admission, $59 \%$ of patients at 10 days and $92.3 \%$ of patients (95\% confidence intervals $78.3-100$ ) at 1 month after stroke [9]. This early period is a critical one for starting rehabilitation [10], and if fatigue delays rehabilitation it may worsen prognosis. There is therefore a need for studies to determine whether admission parameters - in particular changes detectable on neuroimaging - predict fatigue early after stroke and to include both chronic brain changes (atrophy and WML) and acute and chronic stroke lesions.

Although CT is not the most sensitive imaging modality for detecting acute stroke lesions or chronic brain changes, virtually all stroke patients have CT brain images performed for routine clinical care. This study is novel in analysing the relationship between PSF and (i) atrophy and (ii) WML in distinct brain regions, and (iii) acute and previous stroke lesions as detected on routine CT using a simple standardised method. These data are potentially 
useful in determining predictors of PSF in routine clinical practice and may help better understand its nature.

The aim of this study was to identify, in patients with acute stroke, whether features in different brain regions on routine CT brain imaging or routinely collected clinical features predict fatigue at 1 month.

\section{Methods}

\section{Participants}

We recruited participants from a longitudinal cohort study of fatigue after ischaemic or haemorrhagic stroke (the Edinburgh Fatigue after Stroke, EFAS, study) from the Western General Hospital and Royal Infirmary of Edinburgh from July 1, 2009 to June 30, 2011. Exclusion criteria included subarachnoid haemorrhage, severe dysphasia or severe cognitive impairment that would prevent completion of the questionnaires, medical instability and no CT (MRI only). After informed consent, stroke subtype (according to the Oxfordshire Community Stroke Project classification, OCSP) [11], severity (according to the National Institute of Stroke Scale, NIHSS) and patient characteristics were obtained from medical records. Ethical approval for this substudy was obtained in May 2010.

\section{Clinical Assessment}

At recruitment, the Mini-Mental State Examination (MMSE) [12] was performed and prestroke fatigue assessed [13]. At 1 month, participants completed the Fatigue Assessment Scale (FAS) [14] and the Hospital Anxiety and Depression Scale (HADS) [15]. The FAS is a unidimensional fatigue questionnaire consisting of five physical and five mental items each rated on a 5-point scale. Scores on the FAS can range from 10 to 50, with higher scores indicating more fatigue. It has good psychometric properties in different patient populations [14]. The FAS as a measure of fatigue is a validated scale which has been shown to be feasible to administer to patients after stroke [16].

\section{Imaging}

CT brain imaging was performed soon after admission or at the outpatient clinic on a Toshiba 4- or 16-slice scanner until March 2010, and on a 64- or 128-slice scanner thereafter. An experienced neuroradiologist (A.J.F.) rated the CT scans using a standardised pro forma, blind to all other data $[17,18]$. The method recorded the following: (i) cerebral atrophy (none, mild, moderate, severe), (ii) WML (none, mild, moderate, severe), and (iii) presence and site of acute and previous stroke lesions. Central and cortical cerebral atrophy and central and subcortical WML were assessed separately for both sides of the frontal, parietal, temporal and occipital lobes and the cerebellum [19] as well as the brainstem. If patients' CT scans showed no acute stroke lesion, we included these patients in the study and rated the CT scans for atrophy and WML.

\section{Statistics}

Due to the use of short ordinal scales and skewed distribution of some data, we used nonparametric statistics. We investigated the relationship between fatigue score (FAS) and age, gender, stroke severity (NIHSS score), MMSE score, anxiety (HADS-A) and depression (HADS-D) scores, presence of pre-stroke fatigue and OSCP stroke type using Spearman's rho for continuous variables, Mann-Whitney U test (U) for binary variables and the Kruskal Wallis (KW) or $\chi^{2}$ test for categorical variables. To assess the independent contributions of each variable, forward stepwise multiple regression was performed on the FAS (log transformed). 


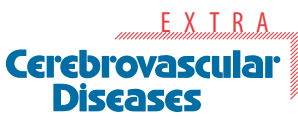

Table 1. Descriptive statistics of participants in study $(\mathrm{n}=107)$

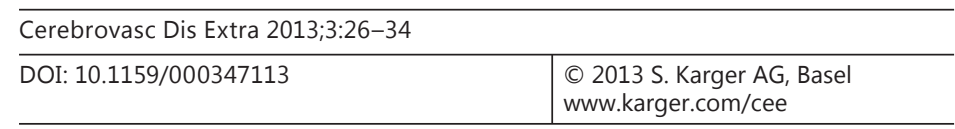

Kutlubaev et al.: CT and Clinical Predictors of Fatigue at One Month after Stroke

\begin{tabular}{lc}
\hline Age, years & $70.5(62-77)$ \\
NIHSS score & $2(1-4)$ \\
MMSE score & $27(25-29)$ \\
FAS score & $23(19-29)$ \\
HADS-A $^{1}$ & $6(3-9)$ \\
HADS-D & 1 \\
Male & $5(3-8)$ \\
Pre-stroke fatigue & $66(62)$ \\
Stroke subtype (OCSP) & $49(45.8)$ \\
$\quad$ TACS & $4(3.5)$ \\
PACS & $47(44)$ \\
LACS & $33(31)$ \\
POCS & $23(21.5)$ \\
\hline \multicolumn{2}{l}{ Values are median (IQR) or n (\%). } \\
\multicolumn{2}{l}{ 'Data collected at 1 month (others collected at baseline). } \\
\hline \multicolumn{2}{l}{}
\end{tabular}

\section{Results}

A total of 161 patients (of 247 approached) were recruited to the EFAS study; of these, 37 did not attend follow-up, 8 had a non-stroke diagnosis, 8 had no CT and 1 declined consent. The remaining 107 patients (66.5\% of recruited patients) were included in this substudy (descriptive data are shown in table 1 and neuroimaging parameters in table 2). There was no difference in baseline characteristics between those included or not in the substudy (data not shown).

Acute visible stroke lesions were present in 40 patients (37.4\%), of whom 31 (30\%) had ischaemic lesion, while $9(8.4 \%)$ had primary intracerebral haemorrhage. Previous vascular lesions were seen in 45 patients (42\%). Some cerebral atrophy of mild or greater severity was present in 84 patients $(77.5 \%)$ and WML of mild or greater severity was present in $54(50.5 \%)$ patients in at least one of the evaluated brain regions. Further, 17 patients $(15.9 \%)$ had no atrophy, WML or acute lesions.

\section{Baseline Characteristics and Fatigue}

FAS scores ranged from 10 to 49 . Women had higher FAS scores [median $=25$; interquartile range $(I Q R)=19-33$ ] than men (median $=22.6$; $I Q R=18-28.25)(U=1,062 ; p=0.05)$. Forty-nine patients $(45.8 \%)$ reported experiencing significant fatigue before stroke. FAS score was not associated with age $(\rho=-0.02 ; p=0.80)$, NIHSS score $(\rho=0.04 ; p=0.68)$, MMSE score $(\rho=-0.005 ; p=0.62)$ or pre-stroke fatigue $(U=1,300 ; p=0.56)$.

\section{Mood and Fatigue}

FAS score was associated with depression $(\rho=0.52 ; \mathrm{p}<0.001)$ and anxiety $(\rho=0.49 ; \mathrm{p}<$ 0.001 ) scores on the HADS at 1 month after stroke.

\section{Neuroimaging Features and Fatigue}

There was no association between fatigue and the presence of any atrophy $(\mathrm{U}=837 ; \mathrm{p}=$ $0.78)$, WML ( $U=1,297 ; p=0.73)$, the presence or absence of an acute stroke lesion $(U=1,247$; $p=0.50)$, the type (infarct/haemorrhage) of the acute lesion if present (KW $=0.29 ; p=0.87$ ) or the presence of old vascular lesions $(U=1,223 ; p=0.49)$. There was also no relationship between the FAS score and the severity of cerebral atrophy (central and cortical) $(U=1,297$; 
Table 2. Descriptive statistics of neuroimaging at baseline $(n=107)$

\begin{tabular}{|c|c|c|c|c|c|}
\hline \multirow[t]{2}{*}{ Location } & \multirow[t]{2}{*}{ Type } & \multicolumn{2}{|l|}{ Right } & \multicolumn{2}{|l|}{ Left } \\
\hline & & $\rho$ & $\mathrm{p}$ & $\rho$ & $\mathrm{p}$ \\
\hline \multicolumn{6}{|l|}{ Atrophy } \\
\hline \multirow[t]{2}{*}{ Frontal lobe } & Central & -0.106 & 0.276 & -0.106 & 0.276 \\
\hline & Cortical & -0.127 & 0.192 & -0.127 & 0.192 \\
\hline \multirow[t]{2}{*}{ Parietal lobe } & Central & -0.137 & 0.160 & -0.137 & 0.160 \\
\hline & Cortical & -0.141 & 0.149 & -0.141 & 0.149 \\
\hline \multirow[t]{2}{*}{ Temporal lobe } & Central & -0.078 & 0.425 & -0.099 & 0.308 \\
\hline & Cortical & -0.198 & 0.041 & -0.104 & 0.066 \\
\hline \multirow[t]{2}{*}{ Occipital lobe } & Central & -0.111 & 0.256 & -0.111 & 0.256 \\
\hline & Cortical & -0.172 & 0.077 & -0.180 & 0.065 \\
\hline \multirow[t]{2}{*}{ Cerebellum } & Central & 0.104 & 0.287 & 0.104 & 0.287 \\
\hline & Cortical & 0.136 & 0.161 & 0.101 & 0.298 \\
\hline Brainstem & & 0.056 & 0.567 & - & - \\
\hline \multicolumn{6}{|l|}{$W M L$} \\
\hline \multirow[t]{2}{*}{ Frontal lobe } & Central & -0.027 & 0.786 & -0.027 & 0.786 \\
\hline & Subcortical & 0.05 & 0.608 & 0.05 & 0.608 \\
\hline \multirow[t]{2}{*}{ Parietal lobe } & Central & -0.036 & 0.710 & -0.041 & 0.675 \\
\hline & Subcortical & -0.028 & 0.776 & -0.031 & 0.749 \\
\hline \multirow[t]{2}{*}{ Temporal lobe } & Central & -0.119 & 0.223 & -0.344 & 0.191 \\
\hline & Subcortical & -0.124 & 0.202 & -0.097 & 0.319 \\
\hline \multirow[t]{2}{*}{ Occipital lobe } & Central & -0.092 & 0.346 & -0.094 & 0.337 \\
\hline & Subcortical & -0.086 & 0.39 & -0.086 & 0.378 \\
\hline Brainstem & & 0.08 & 0.411 & 0.08 & 0.411 \\
\hline
\end{tabular}

Note that ratings of WML and atrophy are very similar (but not identical) in the right and left hemispheres. We found no WML in the cerebellum.

$p=0.73)$ or WML (central and subcortical) $(\mathrm{U}=837 ; \mathrm{p}=0.78)$ assessed both globally and in different regions of the brain (table 3 ).

\section{Stroke Characteristics and Fatigue}

We used the OCSP classification [7] to further explore the possible influence of lesion location because a minority of the patients (37.4\%) had visible acute lesions. Patients with posterior circulation strokes (POCS) had higher FAS scores (median $=28$; IQR $=22-34$ ) than those with lacunar (LACS; median $=24 ; \mathrm{IQR}=17-27.5$ ) or partial (PACS) and total anterior circulation strokes (TACS; median $=23$; IQR $=18-29$ ) analysed together due to the small number of TACS (KW = 6.2; $\mathrm{p}=0.046)$. There was no association between fatigue and side of lesion (POCS excluded as the majority of strokes were subtentorial; $U=1,134 ; p=0.64$ ).

\section{Multivariate Analysis}

Structural CT variables were not significant predictors of fatigue (log FAS) in a linear regression which controlled for age, sex, pre-stroke fatigue, OCSP classification, depression and anxiety. The significant predictors of fatigue were depression $(\beta=0.30 ; p=0.007)$ and anxiety $\left(\beta=0.28 ; p=0.013\right.$; adjusted $R^{2}=0.254$ ). Stroke subtype (according to the OCSP classification) was marginally predictive $(\beta=0.17 ; p=0.05)$ and sex was not statistically significant $(\beta=0.15 ; \mathrm{p}=0.08)$. 
Table 3. Correlations between FAS score and atrophy and WML severity $(n=107)$

\begin{tabular}{|c|c|c|c|c|c|c|c|c|c|}
\hline \multirow[t]{2}{*}{ Location } & \multirow[t]{2}{*}{ Туре } & \multicolumn{4}{|l|}{ Right } & \multicolumn{4}{|l|}{ Left } \\
\hline & & none & mild & $\begin{array}{l}\text { mod- } \\
\text { erate }\end{array}$ & severe & none & mild & $\begin{array}{l}\text { mod- } \\
\text { erate }\end{array}$ & severe \\
\hline \multicolumn{10}{|l|}{ Atrophy } \\
\hline \multirow{2}{*}{ Frontal lobe } & Central & 39 & 39 & 26 & 3 & 39 & 39 & 26 & 3 \\
\hline & Cortical & 35 & 41 & 30 & 1 & 35 & 41 & 30 & 1 \\
\hline \multirow[t]{2}{*}{ Parietal lobe } & Central & 42 & 43 & 23 & 2 & 42 & 40 & 23 & 2 \\
\hline & Cortical & 38 & 45 & 24 & 0 & 38 & 45 & 24 & 0 \\
\hline \multirow[t]{2}{*}{ Temporal lobe } & Central & 53 & 33 & 18 & 3 & 53 & 33 & 18 & 3 \\
\hline & Cortical & 40 & 40 & 25 & 2 & 40 & 40 & 25 & 2 \\
\hline \multirow[t]{2}{*}{ Occipital lobe } & Central & 53 & 36 & 16 & 2 & 53 & 36 & 16 & 2 \\
\hline & Cortical & 50 & 39 & 18 & 0 & 50 & 39 & 18 & 0 \\
\hline \multirow[t]{2}{*}{ Cerebellum } & Central & 101 & 2 & 4 & 0 & 101 & 2 & 4 & 0 \\
\hline & Cortical & 101 & 3 & 3 & 0 & 101 & 3 & 3 & 0 \\
\hline Brainstem & & 105 & 2 & 0 & 0 & & & & \\
\hline \multicolumn{10}{|l|}{$W M L$} \\
\hline \multirow[t]{2}{*}{ Frontal lobe } & Central & 71 & 16 & 20 & 0 & 71 & 17 & 20 & 0 \\
\hline & Subcortical & 72 & 21 & 14 & 0 & 72 & 22 & 13 & 0 \\
\hline \multirow[t]{2}{*}{ Parietal lobe } & Central & 71 & 16 & 20 & 0 & 71 & 17 & 19 & 0 \\
\hline & Subcortical & 74 & 15 & 18 & 0 & 76 & 14 & 17 & 0 \\
\hline \multirow[t]{2}{*}{ Temporal lobe } & Central & 95 & 12 & 0 & 0 & 104 & 13 & 0 & 0 \\
\hline & Subcortical & 96 & 11 & 0 & 0 & 95 & 12 & 0 & 0 \\
\hline \multirow[t]{2}{*}{ Occipital lobe } & Central & 90 & 17 & 0 & 0 & 95 & 12 & 0 & 0 \\
\hline & Subcortical & 90 & 11 & 4 & 2 & 90 & 11 & 3 & 3 \\
\hline Brainstem & & 106 & 1 & 0 & 0 & & & & \\
\hline
\end{tabular}

\section{Discussion}

In this study of 107 patients with acute stroke, features on routine CT (acute or previous vascular lesions, atrophy or WML) did not predict fatigue 1 month after stroke. Clinically diagnosed POCS, female gender and self-reported anxiety and depression were related to PSF.

\section{CT Features}

We found no CT predictors of fatigue at 1 month after stroke. This could be explained by the low sensitivity of CT in detecting cerebral atrophy and WML in comparison to MRI as well as by the low prevalence of acute lesions on CT performed soon after acute stroke. However, the absence of an association between chronic cerebral changes and fatigue in our study does not rule out that these abnormalities could play a role later after stroke, or that they could be detected by more sensitive neuroimaging methodologies such as MRI, although these are not in routine clinical use in all centres. The lack of an association between neuroimaging features and fatigue may be a type II error. There is a potential for bias due to patients who dropped out or died before the follow-up, refused consent or underwent MRI only. However, there was no difference between subjects who were included and those who were excluded, and it is unlikely that associations within cohorts would be substantially different in those included or excluded. 


\section{Clinical Stroke Subtype}

The majority of our patients did not have visible lesions on CT scans, so we used the OCSP classification as an alternative indicator of lesion location. Patients with POCS had higher fatigue scores than those with other stroke subtypes if PACS and TACS were combined. This was done due to the small number of patients with TACS as well as the similarity of the aetiology of PACS and TACS, whereas LACS and POCS may have a distinct aetiology [11]. Previous studies have shown that posterior strokes diagnosed radiologically are associated with fatigue $[1,3,8]$, though prior studies which used the OCSP classification did not find a relationship between stroke type and fatigue [13, 20,21]. Damage to the ascending reticular activating system in the brainstem (supplied by the posterior circulation) may lead to mild impairment in arousal, changes in attention and subsequent development of fatigue. This is a potential area of interest for more detailed neuroimaging studies. Disruption of serotoninergic pathways in the brainstem is another potential mechanism of fatigue after stroke $[1,6]$.

\section{Gender and Mood}

Women after stroke had higher fatigue scores than men, confirming previous findings $[22,23]$. However, it is unclear why women are more vulnerable to PSF than men. Interestingly, chronic fatigue syndrome is also more common in women than in men [24]. Some authors suggested that this could be explained by endocrine and stress-related factors [24].

Patients with higher depression and anxiety scores had higher fatigue scores. Similar results have been reported by previous studies $[22,23]$. However, it is noteworthy that the existing data suggest that, whilst fatigue after stroke is associated with depression, depression does not completely account for fatigue after stroke, and underlying mechanisms of these two phenomena are probably different, as shown by different contributing factors $[1,22]$.

Our study has several strengths: we used routine CT performed soon after clinical presentation with stroke. If we had detected an association between CT features and fatigue at 1 month, this would have had much wider clinical applicability than predictors detected on MRI, which is not routinely performed in all cases or available in all centres. We used validated assessment methods of CT and fatigue features, and this study is novel in rating both acute and chronic stroke lesions as well as both atrophy and WML. We recruited a clinically representative sample of patients with mild-to-moderate stroke. We collected information on fatigue at 1 month after stroke, and it is established that fatigue can be detected this early and start to have an impact on performance and rehabilitation [10].

Some limitations of our study should be acknowledged. It was relatively small (107 participants); however, it was as big as some previously published ones [3]. Publishing negative studies is important in order to avoid publication bias and to ensure that all studies (both negative and positive) can be included in future meta-analyses. The majority of the patients in our cohort had a minor neurological deficit (median NIHSS score $=2$ ). Although we did not aim to recruit patients specifically with mild stroke severity, we had to exclude patients with severe dysphasia as they would have not been able to complete the questionnaires, and it is recognised that patients with more severe strokes are less likely to give consent for research studies [25]. Thus, the results of this study are mostly applicable to patients with mild-to-moderate strokes. We used CT which is significantly less sensitive in both early identification of cerebral ischaemia and detecting WML in comparison to MRI. The finding of an association between fatigue severity and stroke type according to the OCSP classification may be a type I error due to multiple testing and due to the small numbers would be influenced by any small inaccuracy in the OCSP classification. Indeed, if TACS ( $n=4)$ and PACS had not been combined, the result would not have been statistically significant and the OCSP stroke type would not have been included in the multivariate model. 
In summary, we found that fatigue 1 month after stroke was associated with clinical variables and was not associated with neuroimaging features revealed by CT. This suggests that clinical criteria rather than CT features should be used to predict fatigue early after stroke. To establish whether PSF has a structural basis, future studies could investigate the relationship between fatigue and more sensitive neuroimaging techniques (e.g. MRI/DTI) and potential biomarkers (e.g. cortisol). Larger studies could establish whether there is a consistent relationship with stroke subtype. Longer-term follow-up would allow investigation of whether different influences are important at different time points. Establishing what biological mechanisms underlie the development of PSF is essential for the establishment of effective treatment strategies for this common and debilitating consequence of stroke.

\section{Acknowledgements}

M.A.K. was supported by a European Federation of Neurological Societies' Scientific Fellowship. The EFAS study was funded by the Chief Scientist Office of the Scottish Government (CZG/2/387). The neuroimaging substudy of the EFAS study was supported by a grant from The University of Edinburgh Centre for Cognitive Ageing and Cognitive Epidemiology, part of the cross-council Lifelong Health and Wellbeing Initiative (G0700704/84698). Funding from the BBSRC, EPSRC, ESRC and MRC is gratefully acknowledged. S.D.S., J.M.W., G.E.M. and A.M.J.M. are members of The University of Edinburgh Centre for Cognitive Ageing and Cognitive Epidemiology.

\section{References}

1 Kutlubaev MA, Duncan FH, Mead GE: Biological correlates of post-stroke fatigue: a systematic review. Acta Neurol Scand 2012;125:219-227.

2 Naess H, Lunde L, Brogger J: The triad of pain, fatigue and depression in ischemic stroke patients: the Bergen Stroke Study. Cerebrovasc Dis 2012;33:461-465.

-3 Snaphaan L, van der Werf S, de Leeuw FE: Time course and risk factors of post-stroke fatigue: a prospective cohort study. Eur J Neurol 2011;18:611-617.

4 Winward C, Sackley C, Metha Z, Rothwell PM: A population-based study of the prevalence of fatigue after transient ischemic attack and minor stroke. Stroke 2009;40:757-761.

5 Tang WK, Chen YK, Mok V, Chu WC, Ungvari GS, Ahuja AT, Wong KS: Acute basal ganglia infarcts in poststroke fatigue: an MRI study. J Neurol 2010;257:178-182.

-6 Staub F, Bogousslavsky J: Fatigue after stroke: a major but neglected issue. Cerebrovasc Dis 2001;12:75-81.

7 Naess H, Nyland HI, Thomassen L, Aarseth J, Myhr KM: Fatigue at long-term follow-up in young adults with cerebral infarction. Cerebrovasc Dis 2005;20:245-250.

8 Naess H, Lunde L, Brogger J, Waje-Andreassen U: Fatigue among stroke patients on long-term follow-up. The Bergen Stroke Study. J Neurol Sci 2012;312:138-141.

-9 Duncan F, Wu S, Mead GE: Frequency and natural history of fatigue after stroke: a systematic review of longitudinal studies. J Psychosom Res 2012;73:18-27.

10 Langhorne P, Bernhardt J, Kwakkel G: Stroke rehabilitation. Lancet 2011;377:1693-1702.

11 Pittock SJ, Meldrum D, Hardiman O, Thornton J, Brennan P, Moroney JT: The Oxfordshire Community Stroke Project classification: correlation with imaging, associated complications, and prediction of outcome in acute ischemic stroke. J Stroke Cerebrovasc Dis 2003;12:1-7.

12 Folstein MF, Folstein SE, McHugh PR: 'Mini-mental state'. A practical method for grading the cognitive state of patients for the clinician. J Psychiatr Res 1975;12:189-198.

13 Lynch J, Mead G, Greig C, Young A, Lewis S, Sharpe M: Fatigue after stroke: the development and evaluation of a case definition. J Psychosom Res 2007;63:539-544.

14 De Vries J, Michielsen H, Van Heck GL, Drent M: Measuring fatigue in sarcoidosis: the Fatigue Assessment Scale (FAS). Br J Health Psychol 2004; 9:279-291.

15 Zigmond AS, Snaith RP: The Hospital Anxiety and Depression Scale. Acta Psychiatr Scand 1983;67:361-370.

16 Mead G, Lynch J, Greig C, Young A, Lewis S, Sharpe M: Evaluation of fatigue scales in stroke patients. Stroke 2007;38:2090-2095. 
17 Wardlaw JM, Sellar RJ: A simple practical classification of cerebral infarcts on CT and its interobserver reliability. Am J Neuroradiol 1994;15:1933-1939.

18 Brain Research Imaging Centre: Image Analysis Tools. http://www.bric.ed.ac.uk/research/imageanalysis. html (accessed June 4, 2012).

19 Van Swieten JC, Hijdra A, Koudstaal PJ, van Gijn J: Grading white matter lesions on CT and MRI: a simple scale. J Neurol Neurosurg Psychiatry 1990;53:1080-1083.

20 Carlsson GE, Moller A, Blomstrand C: Consequences of mild stroke in persons < 75 years - a 1 year follow-up. Cerebrovasc Dis 2003;16:383-388.

21 Christensen D, Johnsen SP, Watt T, Harder I, Kirkevold M, Andersen G: Dimensions of poststroke fatigue: a two-year follow-up study. Cerebrovasc Dis 2008;26:134-141.

22 Mead GE, Graham C, Dorman P, Bruins SK, Lewis SC, Dennis MS, Sandercock PA; UK Collaborators of IST: Fatigue after stroke: baseline predictors and influence on survival. Analysis of data from UK patients recruited in the International Stroke Trial. PLoS One 2011;6:e16988.

23 Ingles LJ, Eskes AG, Phillips JS: Fatigue after stroke. Arch Phys Med Rehabil 1999;80:173-178.

24 Ranjith G: Epidemiology of chronic fatigue syndrome. Occup Med (Lond) 2005;55:13-19.

25 Kane I, Lindley R, Lewis S, Sandercock P; IST-3 Collaborative Group: Impact of stroke syndrome and stroke severity on the process of consent in the Third International Stroke Trial. Cerebrovasc Dis 2006;21:348-352. 